Conclusion: The CAREGIVERS questionnaire showed to be validated to assess the impact of pediatric rheumatic diseases.

References:

[1] Keppeke, L.d., Molina, J., Miotto e Silva, V.B. et al. Psychological characteristics of caregivers of pediatric patients with chronic rheumatic disease in relation to treatment adherence. Pediatr Rheumatol 16, 63 (2018). https://doi. org/10.1186/s12969-018-0280-7

[2] Cohen EM, Morley-Fletcher A, Mehta DH, Lee YC. A systematic review of psychosocial therapies for children with rheumatic diseases. Pediatr Rheumatol [Internet]. Pediatric Rheumatology; 2017;15(1):6. Available from: http:// ped-rheum.biomedcentral.com/articles/10.1186/s12969-016-0133-1.

[3] Torres-Made, M.D., Peláez-Ballestas, I., García-Rodríguez, F. et al. Development and validation of the CAREGIVERS questionnaire: multi-assessing the impact of juvenile idiopathic arthritis on caregivers. Pediatr Rheumatol 18, 3 (2020). https://doi.org/10.1186/s12969-020-0400-z

[4] Feinstein AR. Clinimetrics. New Haven: Yale University Press, 1987.

Disclosure of Interests: None declared

DOI: 10.1136/annrheumdis-2020-eular.6543

\section{AB0982 SERUM ALBUMIN LEVELS AND DEPRESSION IN JSLE}

S. Ganhão ${ }^{1}$, B. Silva ${ }^{2}$, F. Aguiar ${ }^{3}$, M. Rodrigues ${ }^{3}$, M. Figueiredo-Braga ${ }^{2,4}$, I. Brito ${ }^{2,3}$. ${ }^{1}$ Centro Hospitalar e Universitário de São João, Rheumatology, Oporto, Portugal; ${ }^{2}$ Faculty of Medicine of Oporto's University, Oporto, Portugal; ${ }^{3}$ Centro Hospitalar e Universitário de São João, Young Adult and Pediatric Rheumatology Unit, Oporto, Portugal; ${ }^{4}$ Centro Hospitalar e Universitário de São João, Department of Clinical Neurosciences and Mental Health, Oporto, Portugal

Background: Albumin is a negative acute phase response protein synthesized in the liver, being an important marker of inflammation. Under inflammatory conditions, the transcapillary escape rate of albumin may increase, leading to hypoalbuminaemia. Systemic lupus erythematosus (SLE) is a chronic condition involving multiple organ systems, inducing functional disability and psychological burden responsible for noteworthy depressive symptoms ${ }^{1}$. Depression may be related with psychosocial, environmental and biological factors, disease activity and its severity, age and sex ${ }^{2}$. Several studies show that immune activation and increased concentrations of positive and decreased concentrations of negative acute phase proteins are involved in the pathogenesis of depression ${ }^{3}$. As albumin has the capacity to bind homocysteine, lowered serum albumin levels leads to hyperhomocysteinemia, a well-known risk factor for depression. Moreover, hypoalbuminaemia decrease the availability of tryptophan, an essential amino acid from which the neurotransmitter serotonin is derived, and induce oxidative stress, which further decreases antioxidant levels in people with depression.

Objectives: To assess the association between serum albumin levels and depressive symptoms in juvenile-onset SLE (jSLE) patients.

Methods: A cross-sectional sample of jSLE patients, currently aged $\geq 16$ years, completed a psychosocial assessment including quality of life (SF-36) anxiety and depressive symptoms (HADS) and cognitive assessment (MMSE), between October 2018- May 2019. Local Ethics Committee approved the study. All patients fulfilled both 2012 and 2019 EULAR/ACR classification criteria for SLE. Juvenile-onset was defined as age at diagnosis $<18$ years. Demographics and clinical

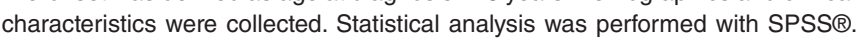
Variables were compared with spearman correlations tests.

Results: 30 jSLE patients were included (90\%female) in the study, with median (min-max) age of 21 (16-35) years, with mean (SD) age of diagnosis of $15.8 \pm 2.1$. Median albumin serum level was 41.7 (16.7-46.3) $\mathrm{g} / \mathrm{dL}$. Psychosocial assessment revealed a mean (SD) score in HADS - Depression of 3.9 (3.3), HADS - Anxiety of 9 (4.3), MMSE of 27.7 (1.8), Physical health SF-36 of 66.8 (9.9) and Mental health SF-36 of 68.9 (17.5). $23.3 \%$ jSLE showed mild cognitive impairment, $63.3 \%$ anxiety and $13.3 \%$ depression. We observed significant inverse linear relationships between serum albumin levels and depressive symptoms score $(p=0.042, \rho=-0.380)$ and with anxiety symptoms score $((p=0.029, \rho=-0.406)$. No significant correlations were detected between albumin serum concentrations and cognitive assessment.

Conclusion: Our findings are consistent with studies previously reporting the potentially protective effect of high serum albumin levels on mental health in different populations. A possible inflammation related aetiology for depression in jSLE patients is highlighted, further explained through the protective roles played by albumin in inflammation, infection, and oxidative damage.

References:

[1] Zhang, L. et al. Prevalence of depression and anxiety in systemic lupus erythematosus: a systematic review and meta-analysis. BMC Psychiatry 17, 70 (2017)

[2] Figueiredo-Braga $M$ et al. Depression and anxiety in systemic lupus erythematosus: The crosstalk between immunological, clinical, and psychosocial factors. Medicine (Baltimore). 2018;97(28)
[3] Livia Ambrus \& Sofie Westling. Inverse association between serum albumin and depressive symptoms among drug-free individuals with a recent suicide attempt, Nordic Journal of Psychiatry, 73:4-5, 229-232

Disclosure of Interests: None declared

DOI: 10.1136/annrheumdis-2020-eular.4608

\section{$\mathrm{AB} 0983$ LIPID METABOLISM AND DISEASE ACTIVITY IN JSLE PATIENTS}

S. Ganhão ${ }^{1}$, A. Mendes ${ }^{2}$, F. Aguiar ${ }^{3}$, M. Rodrigues ${ }^{3}$, I. Brito ${ }^{2,3} \cdot{ }^{1}$ Centro Hospitalar e Universitário de São João, Rheumatology, Oporto, Portugal;

${ }^{2}$ Faculty of Medicine of Oporto's University, Oporto, Portugal; ${ }^{3}$ Centro Hospitalar e Universitário de São João, Young Adult and Pediatric Rheumatology Unit, Oporto, Portugal

Background: Systemic lupus erythematosus (SLE) is an autoimmune systemic disease associated with premature atherosclerosis. Risk factors include dyslipoproteinemia, inflammation, oxidized low-density lipoprotein (LDL), hyperhomocysteinemia and antiphospholipid antibodies. Hyperlipidemic condition is being reported to promote the production of proinflammatory cytokines such as IL-1 $\beta$, IL-6, and IL-27 and lowering blood lipid levels improves the disease. Oxidative stress is elevated, mainly due to mitochondrial dysfunction, further disrupting lipid metabolism. Some drugs also have an impact on lipid profile, such as chronic steroid use, which worsens LDL, HDL, and TG levels.

Objectives: To assess the relationship between lipid profile and disease activity in juvenile SLE (jSLE) patients.

Methods: Retrospective study of jSLE patients, fulfilling both 2012 and 2019 EULAR/ACR classification criteria for SLE. Juvenile-onset was defined as age at diagnosis $<18$ years. Demographics and clinical characteristics were collected. To evaluate the activity of jSLE, the Systemic Lupus Erythematosus Disease Activity Index (SLEDAI) was used. Statistical analysis was performed with SPSS ${ }^{\circ}$. Spearman's rank non-parametric test or Pearson's parametric test were used to assess the bivariate correlation for inflammatory and metabolic variables. $P$ value $<0.05$ was considered significant for all the statistical tests.

Results: 35 patients were included, with current median (min-max) age of 22 (16-35) years, mean (SD) age of diagnosis of 15.8 (2.4) years; $91.4 \%$ female. Median ESR was $19(2-75) \mathrm{mm} / \mathrm{h}$, CRP $1.65(0.1-9.6) \mathrm{mg} / \mathrm{L}$, albumin 41.6 (16.7-46.3) g/L, proteinuria $0.2(0-3) \mathrm{g} / \mathrm{dL}$, leukocyturia $0(0-1362.7) / \mathrm{uL}$, eryth rocyturia $0(0-501.9) / \mathrm{uL}$ and anti-double stranded DNA $89.3(10-800) \mathrm{U} / \mathrm{mL}$. Mean C3 was 102.1 (21.6), C4 17.1 (7.4) mg/dL and creatinine $0.63(0.1) \mathrm{mg}$ dL. Median SLEDAI was 2 (0-12). All were ANA positive, $40 \%$ positive for antinucleossome antibodies, $25.7 \%$ anti-ribossomal $\mathrm{P}$ protein antibody, $11.4 \%$ anti-Sm, 8.6\% autoantibodies against $\beta 2$-glycoprotein I, 8.6\% anti-cardiolipin $14.3 \%$ lupus anticoagulant, $37.1 \%$ anti-SSA and $8.6 \%$ anti-SSB. Articular manifestations were present in $48.6 \%$, mucocutaneous in $77.1 \%$, haematological in $45.7 \%$, lupus nephritis in $42.9 \%$, serositis in $8.6 \%$ and pulmonary interstitial disease in $2.9 \%$. Mean (SD) total cholesterol values (TC) was 165.5 (44.7) $\mathrm{mg} / \mathrm{dL}$ and LDL 94.5 (29.9) $\mathrm{mg} / \mathrm{dL}$. Median high-density lipoprotein was 52 (28-92) and triglycerides (TG) 81.5 (41-253) $\mathrm{mg} / \mathrm{dL}$. Median daily prednisolone dose was $5(0-40) \mathrm{mg}$. $88.6 \%$ were treated with hydroxychloroquine, $31.4 \%$ with mychophenolate mophetil and $14.3 \%$ with azathioprine. TC was negatively correlated with serum albumin $(\mathrm{p}=0.043$, rho $=-378)$ and positively with SLEDAI $(p=0.032$; rho $=0.392)$, proteinuria $(p=0.009$; rho $=0.469)$ and leukocyturia $(p=0.031 ;$ rho $=0.394)$. A positive correlation was found between LDL and proteinuria $(p=0.043$; rho $=0.385)$ and between TG and CRP $(p=0.001$ rho $=0.575$ ). TG were also positively correlated with prednisolone daily dose $(p=0.035$; rho $=0.394)$. Mean LDL was higher in anti-Sm positive patients $(p=0.022)$. No differences were found regarding anti-phospholipids antibodies Nephritic lupus patients had worse lipid metabolism, but this did not reach statistical significance.

Conclusion: In out cohort, increased expression of TC, LDL and TGs is associated with disease activity in SLE. As expected, higher doses of prednisolone also correlated with lipid metabolism.

\section{References:}

[1] Machado D et al. Lipid profile among girls with systemic lupus erythematosus. Rheumatol Int. 2017 Jan;37(1):43-48

Disclosure of Interests: None declared

DOI: 10.1136/annrheumdis-2020-eular.4584

\section{AB0984 BIOLOGICAL THERAPIES IN JUVENILE IDIOPHATIC ARTHRITIS: ARE THERE ANY DIFFERENCES BETWEEN CATEGORIES?}

A. García Fernández ${ }^{1}$, A. Briones-Figueroa ${ }^{1}$, L. Calvo Sanz ${ }^{1}$, Á. AndreuSuárez ${ }^{1}$, J. Bachiller-Corral ${ }^{1}$, A. Boteanu ${ }^{1}$. ${ }^{1}$ Hospital Ramón y Cajal, Rheumatology, Madrid, Spain 